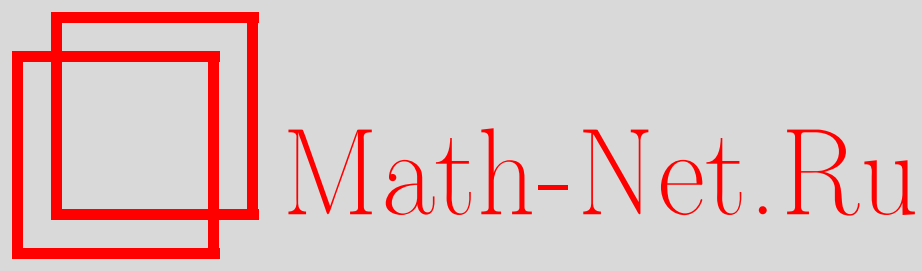

K. Роже, Двойные расширения алгебр Ли типа КацаМуди и их применение к некоторым гамильтоновым системам, ТМФ, 2013, том 174, номер 3, 364-382

DOI: https://doi.org/10.4213/tmf8380

Использование Общероссийского математического портала Math-Net.Ru подразумевает, что вы прочитали и согласны с пользовательским соглашением http://www.mathnet.ru/rus/agreement

Параметры загрузки:

IP : 3.85 .7 .115

26 апреля 2023 г., 15:03:02

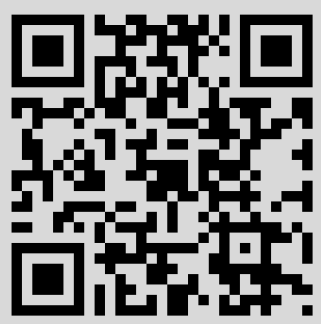




\title{
ДВОЙНЫЕ РАСШИРЕНИЯ АЛГЕБР ЛИ ТИПА КАЦА-МУДИ И ИХ ПРИМЕНЕНИЕ К НЕКОТОРЫМ ГАМИЛЬТОНОВЫМ СИСТЕМАМ
}

\begin{abstract}
Описаны некоторые алгебры Ли типа Каца-Муди, построены их двойные расширения - центральные и полученные с помощью дифференцирований; для некоторых случаев также построены соответствующие группы Ли. Более подробно рассматривается случай алгебры Ли унимодулярных векторных полей. Для построения обобщений некоторых бесконечномерных гамильтоновых систем, подобных магнитогидродинамическим, используется линейная пуассонова структура на их регулярных двойственных пространствах.
\end{abstract}

Ключевые слова: унимодулярные векторные поля, расширения алгебр Ли, гидродинамика, магнитогидродинамика, коприсоединенные орбиты алгебр Ли.

DOI: $10.4213 / \operatorname{tmf} 8380$

\section{1. ВВЕДЕНИЕ}

Прежде всего определим, что мы понимаем под алгебрами Ли типа Каца-Муди: это алгебры Ли $\mathfrak{g}_{\mathcal{A}}$, получаемые взятием тензорного произведения $\mathfrak{g}_{\mathcal{A}}=\mathfrak{g} \otimes \mathcal{A}$ алгебры Ли $\mathfrak{g}$ над полем $\mathbb{K}$ (полем характеристики 0) на некоторую ассоциативную коммутативную $\mathbb{K}$-алгебру $\mathcal{A}$, где скобка естественным образом определяется как $[X \otimes a, Y \otimes b]=[X, Y] \otimes a b$. Если $\mathbb{K}=\mathbb{C}, \mathfrak{g}-$ простая алгебра Ли, а $\mathcal{A}=\mathbb{C}\left[t, t^{-1}\right]-$ алгебра полиномов Лорана, то мы получаем алгебру петель алгебры $\mathfrak{g}$, которую обычно обозначают как $L(\mathfrak{g})$. В более геометрическом случае можно пополнить алгебру и взять $\mathcal{A}=C^{\infty}\left(S^{1}, \mathbb{C}\right)$, что (после подходящего пополнения тензорного произведения) дает $\mathfrak{g}_{\mathcal{A}}=C^{\infty}\left(S^{1}, \mathfrak{g}\right)$ - алгебру Ли токов на $S^{1}$ со значениями в $\mathfrak{g}$.

Соответствующая аффинная нетвистованная алгебра Ли типа Каца-Муди получается путем двойного расширения с центральным членом и с внешним дифференцированием. Сначала построим универсальное чентральное расширение

$$
1 \longrightarrow \mathbb{C} z \longrightarrow \widehat{L(\mathfrak{g})} \longrightarrow L(\mathfrak{g}) \longrightarrow 1,
$$

*Institut Camille Jordan (Laboratoire associé au CNRS UMR 5208), Université Claude Bernard Lyon I, Villeurbanne, France

${ }^{\dagger}$ Ecole Centrale de Lyon, Ecully, France

${ }^{\ddagger}$ Institut National de Sciences Appliquées de Lyon, Villeurbanne, France

${ }^{\S}$ Université de Lyon, Lyon, France. E-mail: roger@math.univ-lyon1.fr 
где $z$ - центральный заряд, порождающий одномерный центр. Затем построим расширение с помощью внешнего дифференцирования:

$$
0 \longrightarrow \widehat{L(\mathfrak{g})} \longrightarrow \widehat{L(\mathfrak{g})} \longrightarrow \mathbb{C} \delta \longrightarrow 0
$$

где генератор $\delta$ фактор-члена представляет собой внешнее дифференцирование $t d / d t$. Замечательный и полезный факт заключается в том, что форму Киллинга $\kappa$ на $\mathfrak{g}$ можно естественным образом расширить на $\widetilde{L(\mathfrak{g})}$ как форму $\mathcal{K}$, которая определяется соотношением $\mathcal{K}(f \otimes x, g \otimes y)=\kappa(x, y) \operatorname{Res}(f d g)$ или, для геометрического случая, соотношением

$$
\mathcal{K}(f \otimes x, g \otimes y)=\kappa(x, y) \int_{S^{1}} f d g .
$$

Более того, мы полагаем, что $\mathcal{K}(z, \delta)=1$, а все остальные члены обращаются в нуль. Эти два расширения являются совместными, их можно объединить в двойное расширение $\widetilde{L(\mathfrak{g})}$, как показано на следующей диаграмме:

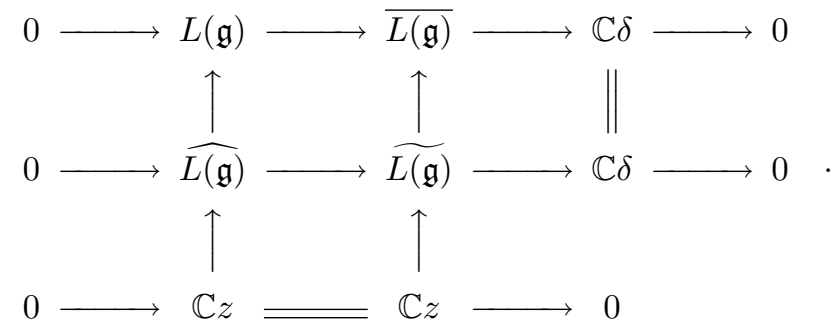

\section{2. ПРЕДВАРИТЕЛЬНЫЕ СВЕДЕНИЯ ИЗ АЛГЕБРЫ}

Напомним некоторые элементарные факты относительно когомологий алгебр Ли, общую теорию и основные приемы можно найти в работе [1]. Для любой алгебры Ли $\mathfrak{g}$ имеется версальное иентральное расширение

$$
0 \longrightarrow H_{2}(\mathfrak{g}) \stackrel{i}{\longrightarrow} \hat{\mathfrak{g}} \stackrel{\pi}{\longrightarrow} \mathfrak{g} \longrightarrow 0 .
$$

Всякий класс когомологий $c \in H^{2}(\mathfrak{g})=\operatorname{Hom}\left(H_{2}(\mathfrak{g}), \mathbb{K}\right)$ индуцирует центральное расширение

$$
0 \longrightarrow \mathbb{K} z \stackrel{i}{\longrightarrow} \tilde{\mathfrak{g}} \stackrel{\pi}{\longrightarrow} \mathfrak{g} \longrightarrow 0 .
$$

Заметим, что как векторное пространство $\tilde{\mathfrak{g}}=\mathfrak{g} \oplus \mathbb{K} z$, а скобка Ли на нем определяется формулой $[x+\lambda z, y+\mu z]=[x, y]+c(x, y) z$. Термин версалъный просто означает, что таким способом можно получить любое центральное расширение. Более того, если $H_{1}(\mathfrak{g})=0$, то расширение (1) является универсалъным в смысле универсального (в данном случае, начального) объекта в категории центральных расширений алгебры $\mathfrak{g}$.

Пусть теперь $M-\mathfrak{g}$-модуль. Элементы пространства $H^{1}(\mathfrak{g}, M)$ классифицируют дифференцирования алгебры $\mathfrak{g}$ со значениями в $M$ по модулю внутренних дифференцирований. Этот результат, в частности, полезен в случае $M=\mathfrak{g}$ с присоединенным представлением, при этом дифференцирование является отображением $\delta: \mathfrak{g} \rightarrow \mathfrak{g}$ таким, что $\delta([X, Y])=[\delta(X), Y]+[X, \delta(Y)]$, в то время как внутреннее дифференцирование задается присоединенным действием некоторого элемента $Z \in \mathfrak{g}$. 
Если пространство $H^{1}(\mathfrak{g}, \mathfrak{g})$ нетривиальное, то можно построить следующее расширение с помощью дифферениирований:

$$
0 \longrightarrow \mathfrak{g} \stackrel{i}{\longrightarrow} \overline{\mathfrak{g}} \stackrel{\pi}{\longrightarrow} H^{1}(\mathfrak{g}, \mathfrak{g}) \longrightarrow 0 .
$$

Для векторных пространств имеем $\overline{\mathfrak{g}}=\mathfrak{g} \oplus H^{1}(\mathfrak{g}, \mathfrak{g})$, а скобка Ли есть

$$
\left[x+\delta_{1}, y+\delta_{2}\right]=[x, y]+\delta_{1}(y)-\delta_{2}(x)+\left[\delta_{1}, \delta_{2}\right]
$$

здесь последний член - коммутатор дифференцирований.

Для обобщения конструкции алгебр Ли типа Каца-Муди можно попытаться построить двойные расширения как [2]

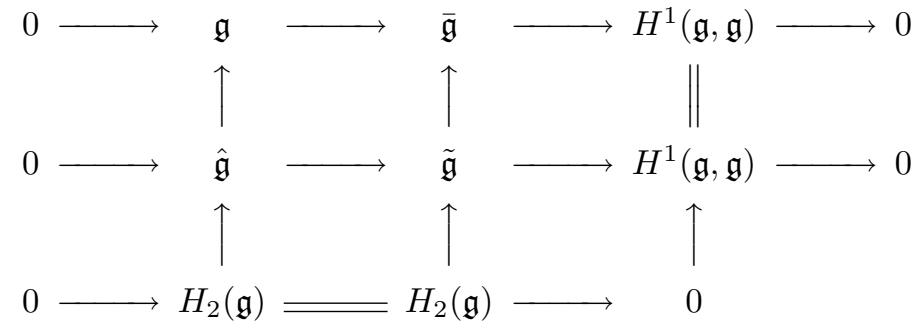

или, выделяя элементы $c \in H^{2}(\mathfrak{g})$ и $\delta \in H^{1}(\mathfrak{g}, \mathfrak{g})$, как

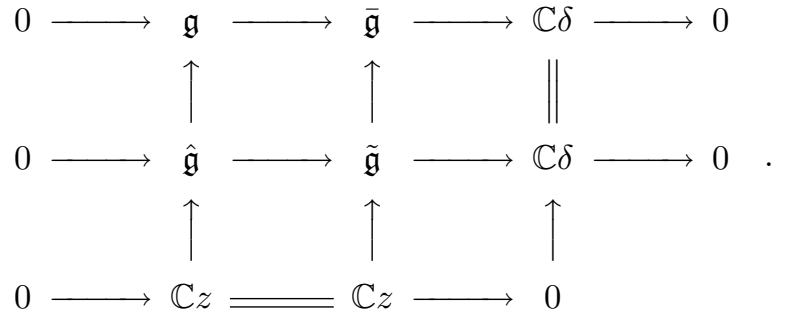

Двойное расширение существует, если и только если выполнено условие совместности

$$
c(\delta X, Y)+c(X, \delta Y)=0, \quad X, Y \in \mathfrak{g} .
$$

Проверьте-ка его!

\section{3. ОСНОВНЫЕ КОНСТРУКЦИИ ДЛЯ АЛГЕБР ЛИ ТИПА КАЦА-МУДИ}

Получим рассмотренные выше конструкции для алгебр Ли $\mathfrak{g}_{\mathcal{A}}$, определенных в разделе 1 , применяя вычисления с использованием гомологий из статей [3]-[5]. Из результатов этих работ следует, что

$$
H_{2}\left(\mathfrak{g}_{\mathcal{A}}\right)=\left(H_{2}(\mathfrak{g}) \otimes \mathcal{A}\right) \oplus\left(B(\mathfrak{g}) \otimes H C_{1}(\mathcal{A})\right) \oplus(?),
$$

где $H C_{1}(\mathcal{A})$ - первая циклическая группа гомологий алгебры $\mathcal{A}$ (см. монографию [6]), а $B(\mathfrak{g})=H_{0}\left(\mathfrak{g}, S^{2}(\mathfrak{g})\right)$ - пространство симметричных коинвариантов степени 2 в $\mathfrak{g}$ (гораздо более популярным является двойственное ему пространство симметричных билинейных инвариантов). Член, обозначенный как (?), обращается в нуль, когда $\mathfrak{g}=[\mathfrak{g}, \mathfrak{g}]$ (алгебра $\mathfrak{g}$ совершенна). Позже мы поясним, как из этих формул в явном виде вывести коциклы. 
Если $\mathcal{A}$ - гладкая алгебра, то

$$
H C_{1}(\mathcal{A})=\Omega^{1}(\mathcal{A}) / d \mathcal{A},
$$

где $\Omega^{1}(\mathcal{A})$ - пространство кэлеровых дифференциалов степени 1 на $\mathcal{A}$ [7]. Типичным примером является $\mathcal{A}=C^{\infty}(\mathcal{X}, \mathbb{R})$ для произвольного компактного ориентируемого многообразия $\mathcal{X}$, так что получаем $H C_{1}(\mathcal{A})=\Omega^{1}(\mathcal{X}) / d \Omega^{0}(\mathcal{X})$. Если $\mathfrak{g}$ - полупростая алгебра, то $H_{2}(\mathfrak{g})=0$, а пространство $B(\mathfrak{g})=H_{0}\left(\mathfrak{g}, S_{2}(\mathfrak{g})\right)$ является одномерным и задается контравариантной версией формы Киллинга (или, если угодно, элементом Казимира). Для внешних дифференцирований можно вывести следующий результат из книги [8]:

$$
H^{1}\left(\mathfrak{g}_{\mathcal{A}}, \mathfrak{g}_{\mathcal{A}}\right)=\left(\operatorname{Id}_{\mathfrak{g}} \otimes \operatorname{Der}(\mathcal{A})\right) \oplus\left(H^{1}(\mathfrak{g}, \mathfrak{g}) \otimes \mathcal{A}\right) .
$$

Здесь $\operatorname{Id}_{\mathfrak{g}}$ обозначает пространство отображений, являющихся скалярными кратными тождественного отображения алгебры $\mathfrak{g}$ в себя.

Если $\mathcal{A}=C^{\infty}(\mathcal{X}, \mathbb{R})$, то $\operatorname{Der}(\mathcal{A})=\operatorname{Vect}(\mathcal{X})$ - алгебра Ли гладких векторных полей на $\mathcal{X}$, а $\mathfrak{g}$ - полупростая алгебра, и тогда мы просто получаем

$$
H^{1}\left(\mathfrak{g}_{\mathcal{A}}, \mathfrak{g}_{\mathcal{A}}\right)=\operatorname{Vect}(\mathcal{X}) .
$$

Векторное поле $\xi \in \operatorname{Vect}(\mathcal{X})$ действует как $L_{\xi}(f \otimes x)=L_{\xi}(f) \otimes x$, поэтому для $\mathcal{A}=C^{\infty}(\mathcal{X}, \mathbb{R})$ и полупростой алгебры $\mathfrak{g}$ можно построить расширения обоих типов:

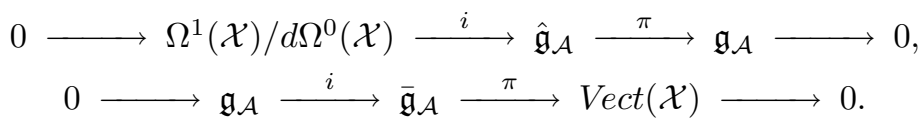

\section{4. ПУАССОНОВЫ СТРУКТУРЫ НА ДВОЙСТВЕННЫХ ПРОСТРАНСТВАХ}

Двойственное пространство $\mathfrak{g}^{*}$ любой алгебры Ли $\mathfrak{g}$ допускает (линейную) пуассонову структуру, симплектические листы которой представляют собой орбиты коприсоединенного действия группы $G$. Это позволяет применить различные аспекты теории Ли к гамильтоновым уравнениям; общее изложение метода орбит приведено в книге [9], а в трудах [10]-[12] описано его применение к динамическим системам. В настоящей работе мы объясним, как модифицируется пуассонова структура на $\mathfrak{g}^{*}$ за счет введения центральных членов или внешних дифференцирований в соответствии с приведенными выше рассуждениями. Пусть $\hat{\mathfrak{g}}$ - центральное расширение, связанное с классом когомологий $c \in H^{2}(\mathfrak{g})$, a $\overline{\mathfrak{g}}-$ расширение алгебры $\mathfrak{g}$ посредством внешнего дифференцирования $\delta$ такие, что выполнено условие совместности (2). Обозначим соответствующее двойное расширение как $\tilde{\mathfrak{g}}$.

Определимся с обозначениями: коприсоединенное действие элемента $X \in \mathfrak{g}$ на $\alpha \in \mathfrak{g}^{*}$ есть $\operatorname{ad}_{X}^{*}(\alpha)$. Теперь вычислим коприсоединенные действия $\widehat{\mathrm{ad}}^{*}, \overline{\mathrm{ad}}^{*}, \mathrm{ad}^{*}$. С элементом $c \in H^{2}(\mathfrak{g})$ можно ассоциировать его коцикл Сурьо $S_{c} \in H^{1}\left(\mathfrak{g}, \mathfrak{g}^{*}\right)$, он получается естественным путем с помощью точной последовательности Кошуля:

$$
0 \longrightarrow \mathfrak{g}^{*} \wedge \mathfrak{g}^{*} \stackrel{i}{\longrightarrow} \mathfrak{g}^{*} \otimes \mathfrak{g}^{*} \stackrel{\pi}{\longrightarrow} S^{2}\left(\mathfrak{g}^{*}\right) \longrightarrow 0 .
$$

Тогда легко найти, что

$$
\widehat{\operatorname{ad}}_{X}^{*}(\alpha, Z)=\left(\operatorname{ad}_{X}^{*}(\alpha)+Z S_{c}(X), 0\right),
$$


где $Z$ дуален центральному члену, который действует тривиально. Таким образом, коцикл Сурьо модифицирует коприсоединенное действие посредством аффинного члена. Аналогично, можно сопоставить $\delta$ дуальный элемент $\delta^{*} \in \operatorname{Hom}\left(\mathfrak{g}^{*}, \mathfrak{g}^{*}\right)$, что дает

$$
\overline{\operatorname{ad}}_{(X, \lambda \delta)}^{*}(\alpha)=\operatorname{ad}_{X}^{*}(\alpha)-\lambda \delta^{*}(\alpha)
$$

(мы ограничили действие $\overline{\mathrm{ad}}^{*}$ на инвариантное подпространство $\mathfrak{g}^{*}$ ). Окончательно для коприсоединенного действия двойного расширения получаем

$$
\widetilde{\operatorname{ad}}_{(X, \lambda \delta)}^{*}(\alpha, Z)=\left(\operatorname{ad}_{X}^{*}(\alpha)+Z S_{c}(X)-\lambda \delta^{*}(\alpha), 0\right)
$$

\section{5. СЛУЧАЙ УНИМОДУЛЯРНЫХ ВЕКТОРНЫХ ПОЛЕЙ}

Пусть $\mathcal{X}$ - ориентируемое многообразие, а $\omega$ - форма объема на нем. Определим алгебру Ли унимодулярных векторных полей на $\mathcal{X}$ следующим образом:

$$
\overline{\operatorname{SVect}}(\mathcal{X})=\left\{X \in \operatorname{Vect}(\mathcal{X}) \mid L_{X}(\omega)=\operatorname{Div}(X) \omega=0\right\} .
$$

Имеем точную последовательность алгебр Ли

$$
0 \longrightarrow \operatorname{SVect}(\mathcal{X}) \stackrel{i}{\longrightarrow} \overline{\operatorname{SVect}}(\mathcal{X}) \stackrel{\pi_{\omega}}{\longrightarrow} H_{d R}^{n-1}(\mathcal{X}) \longrightarrow 0 .
$$

Полагая $\pi_{\omega}(X)=\left[i_{X} \omega\right]$, нетрудно проверить, что $\pi_{\omega}$ является морфизмом алгебры Ли в абелеву алгебру Ли $H_{d R}^{n-1}(\mathcal{X})$. Как следует из результатов работы [13], $S \operatorname{Vect}(\mathcal{X})$ является производным идеалом алгебры $\overline{\operatorname{SVect}}(\mathcal{X})$ и представляет собой совершенную алгебру Ли, известную как алгебра Ли точных унимодулярных векторных полей. Таким образом, абелева алгебра Ли $H_{d R}^{n-1}(\mathcal{X})$ является абелизацией алгебры $\overline{S V e c t}(\mathcal{X})$. Более того, точную последовательность (7) можно интерпретировать в терминах внешних дифференцирований как

$$
H^{1}(\operatorname{SVect}(\mathcal{X}), S \operatorname{Vect}(\mathcal{X}))=H_{d R}^{n-1}(\mathcal{X})
$$

Другими словами, приведенное выше расширение представляет собой расширение посредством дифференцирования [13]-[15].

Алгебра Ли $\operatorname{SVect}(\mathcal{X})$ допускает универсальное центральное расширение [16]-[18]

$$
0 \longrightarrow H_{d R}^{n-2}(\mathcal{X}) \stackrel{i}{\longrightarrow} \widehat{\operatorname{VVect}}(\mathcal{X}) \stackrel{\pi}{\longrightarrow} \operatorname{SVect}(\mathcal{X}) \longrightarrow 0
$$

Здесь универсальный коцикл имеет вид $c(X, Y)=\left[i_{X} i_{Y} \omega\right]$, и поэтому имеется изоморфизм между $H^{2}(S \operatorname{Vect}(\mathcal{X}))$ и $H_{d R}^{n-2}(\mathcal{X}, \mathbb{R})$, а также изоморфизм между $H^{2}(S \operatorname{Vect}(\mathcal{X}))$ и $H_{D R}^{2}(\mathcal{X})$ после применения двойственности Пуанкаре (доказательства приведены в работах [13], [18], а некоторые обобщения для различных геометрических подходов - в книге [16]). Теперь можно собрать два расширения в одно:

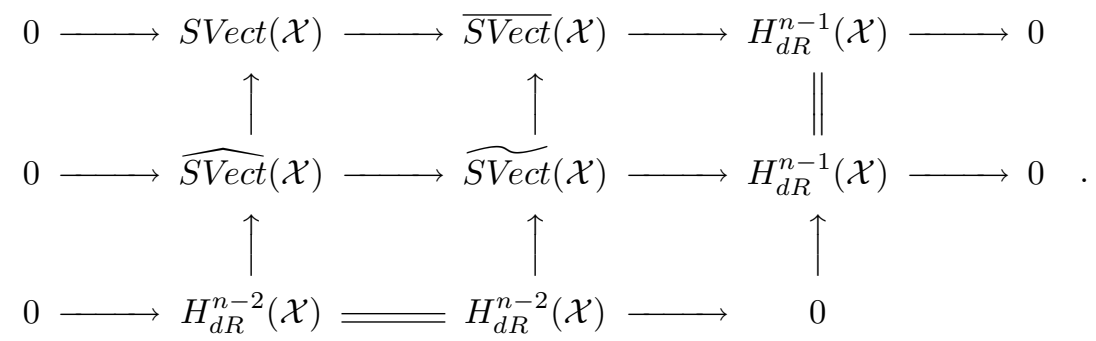


Требуется доказать условие совместности между коциклом и дифференцированиями: пусть $\delta_{W}(X)=[X, W]$ - внешнее дифференцирование алгебры $\operatorname{SVect}(\mathcal{X})$, задаваемое скобкой с некоторым векторным полем $W \in \overline{S V e c t}(\mathcal{X})$. Нетрудно показать, что

$$
c\left(\delta_{W}(X), Y\right)=\left[i_{[X, W]} i_{Y} \omega\right]=\left[i_{X} L_{W} i_{Y} \omega-L_{W} i_{X} i_{Y} \omega\right]=\left[i_{X} L_{W} i_{Y} \omega\right]=\left[i_{[Y, W]} i_{X} \omega\right] .
$$

Таким образом, условие совместности $c\left(\delta_{W}(X), Y\right)+c\left(X, \delta_{W}(Y)\right)=0$ выполнено.

Заметим, что рассмотренные алгебры Ли можно описывать с помощью ковариантных тензоров, используя "музыкальные" изоморфизмы между контравариантными и ковариантными тензорными полями, ассоциированными с формой объема $\omega:$ для $\mathrm{X}$ и $\mathrm{Y}$ из $\overline{\operatorname{SVect}}(\mathcal{X})$, если $i_{X} \omega=\alpha, i_{Y} \omega=\beta$, полагаем $\alpha^{\sharp}=X$ и $\beta^{\sharp}=Y$. Таким образом, получаем отождествления

$$
\begin{aligned}
& Z^{n-1}(\mathcal{X}) \stackrel{\sharp}{\longrightarrow} \overline{\operatorname{SVect}}(\mathcal{X}), \\
& d \Omega^{n-2}(\mathcal{X}) \stackrel{\sharp}{\longrightarrow} \operatorname{SVect}(\mathcal{X}), \\
& \Omega^{n-2}(\mathcal{X}) / d \Omega^{n-3}(\mathcal{X}) \stackrel{\sharp}{\longrightarrow \operatorname{SVect}}(\mathcal{X}) \text {. }
\end{aligned}
$$

При $n=2$ это не что иное, как симплектические и гамильтоновы векторные поля на поверхности, а $\widehat{\operatorname{SVect}}(\mathcal{X})$ легко отождествляется с соответствующей алгеброй Пуассона.

В настоящей работе мы в основном будем полагать, что $n=3$, это соответствует физической модели несжимаемых жидкостей в размерности 3 . В этом случае можно построить некое подобие формы Киллинга на $S \operatorname{Vect}(\mathcal{X})$, известное как инвариант Арнолъда. Пусть $i_{X} \omega=d \alpha, i_{Y} \omega=d \beta$ при $X, Y \in S \operatorname{Vect}(\mathcal{X})$, где $\alpha, \beta \in \Omega^{1}(\mathcal{X})$. Тогда полагаем

$$
\mathcal{J}(X, Y)=\int_{\mathcal{X}} \alpha \wedge d \beta .
$$

Нетрудно проверить, что это билинейное отображение дает корректно определенную, симметричную, невырожденную и инвариантную форму. Более того, ее можно расширить до формы типа формы Киллинга на дважды расширенной алгебре Ли $\widetilde{S \operatorname{Vect}}(\mathcal{X})$; нужно просто использовать двойственность Пуанкаре, чтобы добиться соответствия между $H_{D R}^{1}(\mathcal{X})$ и $H_{D R}^{2}(\mathcal{X})$. Динамическая интерпретация и применения инварианта Арнольда приведены в книге [10].

Прежде чем переходить к приложениям, кратко опишем двойственные пространства этих алгебр Ли. В нашем бесконечномерном случае следует, однако, действовать с оглядкой: поскольку с чисто алгебраическими двойственными пространствами работать невозможно, мы ограничимся линейными отображениями, задаваемыми гладкими мерами плотности. Здесь мы имеем фиксированную форму объема $\omega$, поэтому любая 1-форма $\eta$ следующим образом определяет непрерывное линейное отображение на $\overline{\operatorname{SVect}}(\mathcal{X})$ :

$$
\langle\eta, X\rangle=\int_{\mathcal{X}} \eta(X) \omega .
$$

Если форма $\eta$ точна, то она обращается в нуль тождественно на $\overline{\operatorname{SVect}}(\mathcal{X})$. Отсюда имеем отождествление

$$
\overline{\operatorname{SVect}}(\mathcal{X})_{\text {reg }}^{*}=\Omega^{1}(\mathcal{X}) / d \Omega^{0}(\mathcal{X})
$$


Если форма $\eta$ замкнута, а $X \in S \operatorname{Vect}(\mathcal{X})$, то

$$
\langle\eta, X\rangle=\int_{\mathcal{X}} \eta(X) \omega=-\int_{\mathcal{X}} \eta \wedge i_{X} \omega=-\int_{\mathcal{X}} \eta \wedge d \alpha=\int_{\mathcal{X}} d(\eta \wedge \alpha)=0 .
$$

Тем самым получаем отождествление $S \operatorname{Vect}(\mathcal{X})_{\mathrm{reg}}^{*}=\Omega^{1}(\mathcal{X}) / Z^{1}(\mathcal{X})$. Аналогичные рассуждения дают $\widehat{\operatorname{SVect}}(\mathcal{X})_{\text {reg }}^{*}=Z^{2}(\mathcal{X})$.

Для $n=3$ приведенное выше ковариантное представление унимодулярных векторных полей делает изоморфизмы между алгебрами $\overline{\operatorname{SVect}}(\mathcal{X}), \operatorname{SVect}(\mathcal{X})$ и их регулярными двойственными более естественными, однако инвариант Арнольда $\mathcal{J}$, помимо своего геометрического смысла, дает ad-инвариантный изоморфизм

$$
\mathcal{J}^{\sharp}: \operatorname{SVect}(\mathcal{X})_{\mathrm{reg}}^{*} \rightarrow \operatorname{SVect}(\mathcal{X}),
$$

задаваемый формулой $\mathcal{J}^{\sharp}([u])=d u^{\sharp}$.

\section{6. СТРУИ ВЫСШИХ ПОРЯДКОВ В ГРУППАХ ЛИ И МАГНИТНЫЕ РАСШИРЕНИЯ}

Понятие пролонгации хорошо известно в дифференциальной геометрии благодаря пионерским работам Картана в 20-хгг. прошлого века, идея которых состояла в том, чтобы расширить расслоение реперов на многообразии до расслоений струй реперов высших порядков. С любой группой Ли $G$ можно связать группу ее реперов $k$-го порядка $T^{(k)} G$ с забывающими отображениями $T^{(k)} G \rightarrow T^{\left(k^{\prime}\right)} G$ при $k>k^{\prime}$ и, переходя к проективному пределу, расслоение реперов бесконечного порядка $T^{(\infty)} G$.

Для соответствующих алгебр Ли ситуацию можно описать чисто алгебраически. Пусть $T^{(k)} \mathfrak{g}=\operatorname{Lie}\left(T^{(k)} G\right)$, тогда $T^{(k)} \mathfrak{g}=\mathfrak{g}_{\mathcal{A}_{k}}$, как и выше, где $\mathcal{A}_{k}=\mathbb{K}[t] /\left(t^{k+1}\right)$. Особый смысл имеют два предельных случая: с одной стороны, $T^{(\infty)} \mathfrak{g}=\mathfrak{g} \otimes \mathbb{K} \llbracket t \rrbracket$ является алгеброй Ли бесконечных струй, при этом $\operatorname{Lie}(T G)=T \mathfrak{g}=T^{(1)} \mathfrak{g}=\mathfrak{g}_{\mathcal{A}_{1}}$, а с другой стороны, - алгеброй Ли группы, касательной к $G ;$ обычно $\mathcal{A}_{1}=\mathbb{K}[t] /\left(t^{2}\right)$ называется кольцом дуалъных чисел на $\mathbb{K}$. Более того, существует изоморфизм $T \mathfrak{g}=$ $\mathfrak{g} \ltimes \mathfrak{g}_{\mathrm{Ab}}$, где в правой части полупрямое произведение $\mathfrak{g}$ на себя, рассматриваемую как $\mathfrak{g}$-модуль с абелевой структурой алгебры Ли.

Если $\mathfrak{g}$ допускает форму Киллинга, то $\mathfrak{g}=\mathfrak{g}^{*}$ как $\mathfrak{g}$-модуль, а касательное и кокасательное расслоения на $G$ изоморфны, поэтому $T \mathfrak{g}$ и $T^{*} \mathfrak{g}$ изоморфны как алгебры Ли. Это называется магнитным расширением алгебры $\mathfrak{g}$ (термин был введен Марсденом [19]) по причинам, о которых мы расскажем в следующем разделе. Наконец, форму Киллинга $\kappa$ можно продолжить на $T \mathfrak{g}$, полагая

$$
\kappa(X+t x, Y+t y)=\kappa(X, y)+\kappa(x, Y) .
$$

Этот же прием работает и для $T^{(k)} \mathfrak{g}$, если положить

$$
\kappa\left(\sum_{i=0}^{k} X^{i} t^{i}, \sum_{j=0}^{k} Y^{j} t^{j}\right)=\sum_{i+j=k} \kappa\left(X^{i}, Y^{j}\right) .
$$

Теперь можно вывести конструкции, рассмотренные ранее в разделе 3 (предполагая, что алгебра g совершенна). Имеем $H C^{1} \mathcal{A}_{k}=0$ при $k \neq \infty$ (см. приложение). Отсюда получаем, что

$$
H_{2}\left(T^{(k)} \mathfrak{g}\right)=H_{2}(\mathfrak{g}) \otimes \mathcal{A}_{k}=H^{2}(\mathfrak{g}) \otimes \mathcal{A}_{k}^{\prime} .
$$


Нетрудно заметить, что $\operatorname{Der}\left(\mathcal{A}_{k}\right)=I(d / d t)$, где $I=(t)$ - максимальный идеал локального кольца $\mathcal{A}_{k}, \operatorname{Der}\left(\mathcal{A}_{k}\right)$ - нильпотентная алгебра Ли, в частности, алгебра $\operatorname{Der}\left(\mathcal{A}_{1}\right)$ одномерная и порождена элементом $t d / d t$. Отсюда имеем

$$
H^{1}\left(T^{(k)} \mathfrak{g}, T^{(k)} \mathfrak{g}\right)=\left(\operatorname{Id}_{\mathfrak{g}} \otimes \operatorname{Der}\left(\mathcal{A}_{k}\right)\right) \oplus\left(H^{1}(\mathfrak{g}, \mathfrak{g}) \otimes \mathcal{A}_{k}\right) .
$$

Если $k=\infty$, то $\operatorname{Der}\left(\mathcal{A}_{\infty}\right)=\mathbb{K} \llbracket t \rrbracket d / d t$ - алгебра Ли формальных векторных полей от одной переменной, при этом $H C^{1} \mathcal{A}_{\infty}=\Omega^{1}(\mathbb{K} \llbracket t \rrbracket) / d \mathbb{K} \llbracket t \rrbracket=0$, откуда следует, что

$$
\begin{aligned}
H_{2}\left(T^{(\infty)} \mathfrak{g}\right) & =H_{2}(\mathfrak{g}) \otimes \mathbb{K} \llbracket t \rrbracket \\
H^{1}\left(T^{(\infty)} \mathfrak{g}, T^{(\infty)} \mathfrak{g}\right) & =\left(\operatorname{Id}_{\mathfrak{g}} \otimes \mathbb{K} \llbracket t \rrbracket \frac{d}{d t}\right) \oplus\left(H^{1}(\mathfrak{g}, \mathfrak{g}) \otimes \mathcal{A}_{\infty}\right) .
\end{aligned}
$$

Теперь рассмотрим некоторые частные случаи и вычислим в явном виде соответствующую модифицикацию пуассоновых структур на двойственных пространствах, ассоциированную с центральным членом или с дифференцированием, как это было сделано ранее в разделе 4. Прежде всего заметим, что $\mathcal{A}_{k}^{\prime}$ и $\mathcal{A}_{k}$ изоморфны как $\mathbb{K}$-векторные пространства; обозначим через $\tau$ переменную, двойственную к $t$.

Если группа $G$ полупростая, то $H^{1}\left(T^{(1)} \mathfrak{g}, T^{(1)} \mathfrak{g}\right)=\operatorname{Id}_{\mathfrak{g}} \otimes \mathbb{R}(\delta)$. Действие последнего дифференцирования в явном виде записывается как $\delta(X+t x)=t x$, и на двойственном уровне $\delta^{*}(A+\tau a)=\tau a$. Тогда коприсоединенное действие модифицируется следующим образом:

$$
\overline{\operatorname{ad}}_{(X+t x, \lambda \delta)}^{*}(A+\tau a)=\operatorname{ad}_{X}^{*}(A)+\operatorname{ad}_{x}^{*}(a)+\tau\left(\operatorname{ad}_{X}^{*}(a)-\lambda a\right) .
$$

Если группа $G$ является полупростой, то $H_{2}\left(T^{(1)} \mathfrak{g}\right)=0$, поэтому приведенная выше формула дает единственную возможную модифицикацию - мы получили нечто вроде "игрушечной модели".

Теперь рассмотрим случай $\mathfrak{g}=S \operatorname{Vect}(\mathcal{X})$ : из приведенных выше вычислений следует, что

$$
H^{2}\left(T^{1} \operatorname{SVect}(\mathcal{X})\right)=H^{2}(\operatorname{SVect}(\mathcal{X})) \otimes \mathcal{A}_{1}^{\prime}=H_{d R}^{2}(\mathcal{X}) \otimes \mathcal{A}_{1}^{\prime} .
$$

Из свойств универсального центрального расширения $S \operatorname{Vect}(\mathcal{X})$, определенного выше, немедленно получаем центральный коцикл, ассоциированный с классом когомологий $[H] \in H_{d R}^{2}(\mathcal{X})$ для $H \in \Omega^{2}(\mathcal{X})$. Имеем

$$
c_{[H]}(X, Y)=\int_{\mathcal{X}} H \wedge i_{X} i_{Y} \omega=\int_{\mathcal{X}} H(X, Y) \omega .
$$

Эквивалентная формула такова: рассмотрим подмногообразие $N \in \mathcal{X}$ коразмерности 2 такое, что его класс когомологий $[N] \in H_{n-2}(\mathcal{X})$ является пуанкаре-двойственным к $[H]$, тогда [14]

$$
c_{[N]}(X, Y)=\int_{[N]} \omega(X, Y) .
$$

Теперь пусть $[H]+\tau[h] \in H_{d R}^{2}(\mathcal{X}) \otimes \mathcal{A}_{1}^{\prime}$ для $T^{1} S \operatorname{Vect}(\mathcal{X})$, тогда

$$
c_{[H]+\tau[h]}(X+\tau x, Y+\tau y)=\int_{\mathcal{X}}(H(X, Y)+h(x, Y)+h(X, y)) \omega .
$$

Из этих формул нетрудно вывести выражение для соответствующего коцикла Сурьо. Напомним, что мы определили регулярное двойственное пространство $S \operatorname{Vect}(\mathcal{X})_{\mathrm{reg}}^{*}=$ $\Omega^{1}(\mathcal{X}) / Z^{1}(\mathcal{X})$, отсюда $T^{1} S \operatorname{Vect}(\mathcal{X})_{\text {reg }}^{*}=\left(\Omega^{1}(\mathcal{X}) / Z^{1}(\mathcal{X})\right) \otimes \mathcal{A}_{1}^{\prime}$. Получаем

$$
S_{[H]+\tau[h]} \in H^{1}\left(T^{1} \operatorname{SVect}(\mathcal{X}),\left(\Omega^{1}(\mathcal{X}) / Z^{1}(\mathcal{X})\right) \otimes \mathcal{A}_{1}^{\prime}\right),
$$


что вытекает из формулы $S_{[H]+\tau[h]}(X+t x)=\left[i_{X} H+i_{x} h\right]+\tau\left[i_{X} h\right]$ (здесь в правой части равенства скобки обозначают классы эквивалентности 1-форм по модулю замкнутых 1-форм). Теперь можно выписать в явном виде коприсоединенное действие в расширенной алгебре Ли (см. раздел 4, формула (5)):

$$
\widehat{\operatorname{ad}}_{X+t x}^{*}(A+\tau a, Z+\tau z)=\operatorname{ad}_{X}^{*}(A)+\operatorname{ad}_{x}^{*}(a)+Z\left[i_{X} H+i_{x} h\right]+\tau\left(\operatorname{ad}_{X}^{*}(a)+z\left[i_{X} h\right]\right),
$$

где элемент $Z+\tau z$ соответствует центральному заряду, ассоциированному с коциклом

$[H]+\tau[h]$.

Получим теперь члены, соответствующие внешним дифференцированиям: для $\mathfrak{g}=\operatorname{SVect}(\mathcal{X})$ вычисления дают

$$
\begin{gathered}
H^{1}\left(T^{(1)} \operatorname{SVect}(\mathcal{X}), T^{(1)} S \operatorname{Vect}(\mathcal{X})\right)= \\
=\left(\operatorname{Id}_{S \operatorname{Vect}(\mathcal{X})} \otimes \mathbb{R}(\delta)\right) \oplus\left(H^{1}(\operatorname{SVect}(\mathcal{X}), S \operatorname{Vect}(\mathcal{X})) \otimes \mathcal{A}_{1}\right), \\
H^{1}(\operatorname{SVect}(\mathcal{X}), S \operatorname{Vect}(\mathcal{X}))=H_{d R}^{n-1}(\mathcal{X}) .
\end{gathered}
$$

Отсюда окончательно получаем

$$
\begin{aligned}
& H^{1}\left(T^{(1)} \operatorname{SVect}(\mathcal{X}), T^{(1)} \operatorname{SVect}(\mathcal{X})\right)=\left(\operatorname{Id}_{S \operatorname{Vect}(\mathcal{X})} \otimes \mathbb{R}(\delta)\right) \oplus\left(H_{d R}^{n-1}(\mathcal{X}) \otimes \mathcal{A}_{1}\right), \\
& H^{1}\left(T^{(1)} \overline{\operatorname{SVect}}(\mathcal{X}), T^{(1)} \overline{\operatorname{SVect}}(\mathcal{X})\right)=\left(\operatorname{Id}_{S \operatorname{Vect}(\mathcal{X})} \otimes \mathbb{R}(\delta)\right) .
\end{aligned}
$$

Теперь можно выписать коприсоединенное действие, модифицированное указанными внешними дифференцированиями (см. формулу (6)):

$\overline{\operatorname{ad}}_{(X+t x, \lambda \delta+U+t W)}^{*}(A+\tau a)=\operatorname{ad}_{X}^{*}(A)+\operatorname{ad}_{x}^{*}(a)-U^{*}(A)+\tau\left(\operatorname{ad}_{X}^{*}(a)-U^{*}(a)-W^{*}(A)-\lambda a\right)$.

Здесь $U+t W \in H_{d R}^{n-1}(\mathcal{X}) \otimes \mathcal{A}_{1}$. Напомним, что в данном контексте группа когомологий $H_{d R}^{n-1}(\mathcal{X})$ появляется как фактор $\overline{S V e c t}(\mathcal{X})$ по $S \operatorname{Vect}(\mathcal{X})$, поэтому $U$ и $W$ можно рассматривать как классы векторных полей в $\overline{\operatorname{SVect}}(\mathcal{X})$ по модулю $\operatorname{SVect}(\mathcal{X})$.

В разделе 5 мы получили геометрическое описание регулярных двойственных пространств, на основе которого $A$ и $а$ можно отождествить с классами 1-форм $[u]$ и $[b]$ в $\Omega^{1}(\mathcal{X}) / Z^{1}(\mathcal{X})$, а описанное выше коприсоединенное действие сделать более явным, отождествляя его с производной Ли на дифференциальных формах. Получаем

$$
\overline{\operatorname{ad}}_{(X+t x, \lambda \delta+U+t W)}^{*}([u]+\tau[b])=\left[L_{X} u+L_{x} b-L_{U} u\right]+\tau\left[L_{X} b-L_{U} b-L_{W} u-\lambda b\right],
$$

а для рассмотренного выше центрального расширения имеем

$$
\widehat{\operatorname{ad}}_{X+t x}^{*}([u]+\tau[b], Z+\tau z)=\left[L_{X} u+L_{x} b\right]+Z\left[i_{X} H+i_{x} h\right]+\tau\left(\left[L_{X} b\right]+z\left[i_{X} h\right]\right) .
$$

\section{7. ГРУППЫ ДИФФЕОМОРФИЗМОВ, АССОЦИИРОВАННЫЕ С РАСШИРЕНИЯМИ}

Не всегда можно интегрировать бесконечномерную алгебру Ли до группы, даже в банаховом случае. В нашем случае группа объема, сохраняющая диффеоморфизмы $\overline{S \operatorname{Diff}}(\mathcal{X})$, является группой Ли типа Фреше, которая интегрирует алгебру Ли $\overline{S V e c t}(\mathcal{X})$ (в слабом смысле). При этом отображение алгебр Ли

$$
\overline{\operatorname{SVect}}(\mathcal{X}) \stackrel{\pi_{\omega}}{\longrightarrow} H_{d R}^{n-1}(\mathcal{X}) \longrightarrow 0
$$

интегрируется до группового гомоморфизма

$$
\widetilde{\operatorname{SDiff}}(\mathcal{X}) \stackrel{\widetilde{\Pi}_{\omega}}{\longrightarrow} H_{d R}^{n-1}(\mathcal{X})
$$


где $\widetilde{S D i f f}(\mathcal{X})$ представляет собой универсальное накрытие связной компоненты единицы $\overline{\operatorname{SDiff}}(\mathcal{X})_{0} \subset \overline{\operatorname{SDiff}}(\mathcal{X})$.

Групповой гомоморфизм $\widetilde{\Pi}_{\omega}$, называемый потоком, впервые был определен В. Терстоном (неопубликованная работа, подробности приведены в [14], [20]). Пусть $\Lambda_{\omega}=\widetilde{\Pi}_{\omega}\left(\pi_{1}(\overline{S D i f f}(\mathcal{X}))\right)$ - образ фундаментальной группы пространства $\overline{S D i f f}(\mathcal{X})$, тогда $\widetilde{\Pi}_{\omega}$ отправляется в фактор как

$$
\overline{\operatorname{SDiff}}(\mathcal{X})_{0} \stackrel{\Pi_{\omega}}{\longrightarrow} H_{d R}^{n-1}(\mathcal{X}) / \Lambda_{\omega} .
$$

Можно показать, что образ $\Lambda_{\omega}$ дискретный, а потому фактор-группа является хаусдорфовой, если форма объема $\omega$ имеет целочисленные периоды (подробности и различные геометрические интерпретации также приведены в [14], [20]). Теперь нам нужно решить проблему интегрирования центральных расширений, для чего мы снова воспользуемся результатами работы [14]. Гомоморфизм потока можно обобщить на дифференциальные формы любой степени, и для 2-коциклов это аналогично тому, как мы сделали в предыдущем разделе для коцикла Сурьо. А именно, пусть $c$ - некий 2-коцикл на $\overline{S V e c t}(\mathcal{X})$, а ассоциированный с ним инфинитезимальный поток есть $s_{c}: \overline{S V e c t}(\mathcal{X}) \rightarrow \overline{S V e c t}(\mathcal{X})_{\text {reg }}^{*}$, который определяется как $s_{c}(X)=i_{X} c$. Его можно интегрировать непосредственно на универсальной накрывающей группе как

$$
\mathcal{S}_{c}: \widetilde{\operatorname{Siff}}(\mathcal{X}) \rightarrow \overline{\operatorname{SVect}}(\mathcal{X})_{\mathrm{reg}}^{*}, \quad \mathcal{S}_{c}([\gamma])(X)=-\int_{\gamma} i_{X^{*}} c^{*},
$$

где $[\gamma]$ - путь, начинающийся в $\overline{\operatorname{SDiff}}(\mathcal{X}), X^{*}$ - правое инвариантное векторное поле на группе, ассоциированное с $X$, а $c^{*}$ - левая инвариантная форма на группе, ассоциированная с коциклом $c$ (подробности можно найти в работе [14]).

Если теперь ограничить гомоморфизм потока на фундаментальную группу, то получим $\mathcal{S}_{c}: \pi_{1}(S \operatorname{Diff}(\mathcal{X})) \rightarrow H_{1}(\overline{\operatorname{SVect}}(\mathcal{X}))=H_{n-1}(\mathcal{X})$. Если коцикл $c=c_{[N]}$ ассоциирован с $(n-2)$-мерным многообразием $N \subset \mathcal{X}$, как и ранее, то поток удовлетворяет условию

$$
\mathcal{S}_{c_{[N]}}: \pi_{1}(S \operatorname{Diff}(\mathcal{X})) \rightarrow H_{1}(\overline{S \operatorname{Vect}}(\mathcal{X}))=H_{n-1}(\mathcal{X})
$$

и задается как $\mathcal{S}_{c_{[N]}}\left(\left[\gamma_{t}\right]\right)=[\gamma N] \in H_{n-1}(\mathcal{X})$, где $[\gamma N]-(n-1)$-цикл в $\mathcal{X}$, который определяется как $[\gamma N](t, x)=\gamma_{t}(x) \in \mathcal{X}$ для $(t, x) \in[0,1] \times N[14]$.

Еще одно препятствие к интегрируемости алгебры Ли до группы Ли зависит от гомотопической группы степени два группы диффеоморфизмов. Пусть $G$ - одна из рассмотренных выше групп диффеоморфизмов, сохраняющих объем. Поставим 2-коциклу $c$ в соответствие его гомоморфизм с периодом

$$
\operatorname{per}_{c}: \pi_{2}(G) \rightarrow_{x} \mathbb{R}, \quad \operatorname{per}_{c}([\sigma])=\int_{S^{2}} \sigma^{*}\left(c^{*}\right),
$$

где $\sigma: S^{2} \rightarrow G$ представляет гомотопический класс, а $c^{*}-$ как и ранее, лево-инвариантная 2-форма на $G$, ассоциированная с коциклом $c$. Тогда группа периодов коцикла $c$ есть $\Gamma_{c}=\operatorname{Im}\left(\operatorname{per}_{c}\right)$.

Окончательный результат имеет следующий вид (теорема 5 из статьи [14]).

Теорема 1. Если группа $\Gamma_{c}$ дискретна и гомоморфизм потока $S_{c}$ обращается в нуль, то имеется центральное расширение групп Ли

$$
1 \longrightarrow \mathbb{R} / \Gamma_{c} \longrightarrow \widehat{G} \longrightarrow G \longrightarrow 1
$$

которое интегрирует расширение алгебр Ли, соответствующее коциклу с. 
Если коцикл $c=[H]$ ассоциирован с 2-формой $H$, то, как нетрудно видеть, группа $\Gamma_{[H]}$ содержится в группе периодов формы $H$. Поэтому если периоды формы $H$ целочисленные, то $\Gamma_{[H]}$ дискретна; это имеет место, в частности, когда $c=c_{[N]}$ для подмногообразия $N \subset \mathcal{X}$.

В итоге можно заключить, что за интегрирование различных расширений алгебр Ли, рассмотренных в разделе 5 , нужно заплатить некую цену топологической природы: группы $\Gamma_{c}$ и $\Lambda_{\omega}$ должны быть дискретными, а поток $S_{c}$ должен обращаться в нуль. Разумеется, вычисление гомотопических групп $\pi_{1}(G)$ и $\pi_{2}(G)$ для наших групп диффеоморфизмов $G$ представляет собой сложную топологическую задачу, про которую, насколько мы знаем, известно не так много. Мы можем удостовериться в тривиальности потока $S_{c}$ в очевидных случаях: поскольку группа $S \operatorname{Vect}(\mathcal{X})$ совершенна [13], ее 1-гомологии обращаются в нуль. Следовательно, гомоморфизм потока обращается в нуль для $\operatorname{SD} i f f(\mathcal{X})$, как и в случае универсального накрытия $\widehat{\operatorname{SDiff}}(\mathcal{X})$, фундаментальная группа которого тривиальна по определению:

$$
\begin{aligned}
& 1 \longrightarrow \mathbb{R} / \Gamma_{c_{[N]}} \longrightarrow \widehat{\operatorname{SDiff}}(\mathcal{X}) \longrightarrow \widehat{\operatorname{SDiff}}(\mathcal{X}) \longrightarrow \\
& 1 \longrightarrow \Gamma_{c_{[N]}} \longrightarrow \widehat{\operatorname{SDiff}}(\mathcal{X}) \longrightarrow 1
\end{aligned}
$$

Эти центральные расширения соответствуют заданию вертикальных стрелок на диаграмме (8), поскольку универсальное накрытие $\widehat{\operatorname{SDiff}}(\mathcal{X})$ интегрирует алгебру Ли $\overline{S V e c t}(\mathcal{X})$. В действительности, о расширении (10) можно сказать больше. Пусть $K_{c}$ - ядро гомоморфизма потока, тогда можно центральным образом продолжить фактор $\widehat{\widehat{\operatorname{SDiff}}}(\mathcal{X}) / K_{c}$, что дает наилучшее возможное уточнение сказанного выше. Если, более того, форма объема $\omega$ интегральная, то мы получаем группу, расширение которой интегрирует (7):

$$
1 \longrightarrow \operatorname{SDiff}(\mathcal{X}) \longrightarrow \overline{\operatorname{Siff}}(\mathcal{X})_{0} \stackrel{\Pi_{\omega}}{\longrightarrow} H_{d R}^{n-1}(\mathcal{X}) / \Lambda_{\omega} \longrightarrow 1 .
$$

Эта последовательность является точной последовательностью абелизации, так что коммутаторы $\left[\overline{S \operatorname{Diff}}(\mathcal{X})_{0}, \overline{\operatorname{SDiff}}(\mathcal{X})_{0}\right] \subset \operatorname{SDiff}(\mathcal{X})$. В действительности эти две подгруппы совпадают с точностью до, возможно, некоторого дискретного фактора.

Здесь необходимо отметить, хоть он нам и не потребуется, очень эффектный аргумент, который был использован в работе [14] с целью построения центральных расширений этих групп, для чего применялось геометрическое описание сингулярного двойственного пространства $\overline{S V e c t}(\mathcal{X})_{\operatorname{sing}}^{*}$ с помощью изотопических классов подмногообразий коразмерности 2 (обобщенных узлов) и их геометрического квантования.

Наш последний шаг в построении групп не представляет серьезных трудностей: касательные или кокасательные алгебры Ли легко интегрировать, так как $T^{(1)} \operatorname{SVect}(\mathcal{X}), T^{(1)} \overline{S V e c t}(\mathcal{X})$ суть алгебры Ли групп

$$
T^{(1)} \operatorname{SDiff}(\mathcal{X})=\operatorname{SDiff}(\mathcal{X}) \ltimes \operatorname{SVect}(\mathcal{X}), \quad T^{(1)} \overline{\operatorname{SDiff}}(\mathcal{X})=\overline{\operatorname{SDiff}}(\mathcal{X}) \ltimes \overline{\operatorname{SVect}}(\mathcal{X})
$$

соответственно. Дополнительное дифференцирование $\operatorname{Id}_{S \operatorname{Vect}(\mathcal{X})} \otimes \mathbb{R}(\delta)$ без особых трудностей интегрируется до однопараметрической группы автоморфизмов пространства $T^{(1)} \overline{S D i f f}(\mathcal{X})$ : мы имеем $F_{s}(\phi+t \xi)=\phi+t e^{s} \xi$. Наконец, центральные расширения $T^{(1)} \operatorname{SDiff}(\mathcal{X})$ и $T^{(1)} \overline{S \operatorname{Diff}}(\mathcal{X})$ легко получить из касательных групп $(9),(10)$. 


\section{8. ДИНАМИЧЕСКАЯ ИНТЕРПРЕТАЦИЯ И ПРИЛОЖЕНИЕ К МАГНИТОГИДРОДИНАМИКЕ}

Начиная с пионерских работ Арнольда, выполненных в 60-х гг. прошлого века, хорошо известно, что группа Ли сохраняющих объем диффеоморфизмов $\overline{S \operatorname{Diff}}(\mathcal{X})$ представляет собой конфигурационное пространство для динамики несжимаемой жидкости с фиксированной формой объема $\omega$; векторное поле скоростей живет в его алгебре Ли $\mathfrak{g}=S \operatorname{Vect}(\mathcal{X})$, а уравнение Эйлера дает $\dot{\xi}+\nabla_{\xi} \xi+\nabla p=f$. Здесь $\xi \in \mathfrak{g}-$ поле скоростей в эйлеровом описании, $p$ - поле давления в жидкости, а $f$ - поле внешних сил, представляющее все возможные виды внешнего воздействия, например электромагнитные или, возможно, неабелевы калибровочные взаимодействия.

Следуя подходу книги [10], напомним, как можно сделать это уравнение гамильтоновым на регулярном двойственном пространстве $S \operatorname{Vect}(\mathcal{X})_{\mathrm{reg}}^{*}=\Omega^{1}(\mathcal{X}) / Z^{1}(\mathcal{X})$. Выберем метрику на $\mathfrak{g}$ не зависящей от формы объема и обозначим скалярное произведение просто точкой. Тогда можно построить оператор инериии $I: \mathfrak{g} \rightarrow \mathfrak{g}^{*}$, естественным образом задаваемый формулой $\langle I(\xi), \eta\rangle=\xi \cdot \eta$, где скобки $\langle\cdot, \cdot\rangle$ обозначают, как обычно, спаривание между векторным пространством и его двойственным. Такой изоморфизм зависит от физики задачи, можно называть его динамическим изоморфизмом в отличие от кинематического изоморфизма $\mathcal{J}^{\sharp}$, определенного ранее (подробности приведены в книге [10] и статье [21], где использовался аналогичный подход к решению несколько другой задачи). Теперь мы может определить энергию на $\mathfrak{g}$ и двойственно на $\mathfrak{g}^{*}$ как

$$
H(\xi)=\frac{1}{2}\|\xi\|^{2}=\frac{1}{2}\langle I(\xi), \xi\rangle, \quad H([u])=\frac{1}{2}\left\langle[u], I^{-1}[u]\right\rangle
$$

соответственно. Тогда можно переписать приведенное выше уравнение Эйлера как гамильтоново уравнение $[\dot{u}]+L_{I^{-1}[u]}[u]=0$ на пуассоновом многообразии $\mathfrak{g}^{*}$ (без внешних сил). В терминах коприсоединенного действия получается уравнение эквивалентного вида $[\dot{u}]+\operatorname{ad}_{I^{-1}[u]}^{*}[u]=0$, которое также, очевидно, является гамильтоновым. Давление при этом включается в класс $[u]$ 1-формы $u$ по модулю точных форм [10].

Теперь рассмотрим магнитогидродинамику (МГД). Пусть жидкость несет электрические заряды, на которые действуют лоренцевы силы, и создает свое собственное поле посредством движущихся зарядов. На самом деле мы рассмотрим частный случай, когда электрическим полем можно пренебречь, а магнитное поле заморожено в жидкости, подробности можно найти в книге [22]. Таким образом, как материальное поле $\xi$, так и магнитное поле $B$ в $S \operatorname{Vect}(\mathcal{X})$ допускают следующие эволюционные уравнения:

$$
\dot{\xi}+\nabla_{\xi} \xi+\nabla p-\operatorname{rot}(B) \wedge B=0, \quad \dot{B}+[\xi, B]=0 .
$$

Теперь можно видеть, что конфигурационное пространство для МГд представляет собой полупрямое произведение $\operatorname{SDiff}(\mathcal{X}) \ltimes \Omega^{1}(\mathcal{X}) / Z^{1}(\mathcal{X})$, а соответствующая алгебра Ли является магнитным расширением $T^{*} S \operatorname{Vect}(\mathcal{X})$. В книге [10] приведена гамильтонова интерпретация уравнений МГД на алгебре, двойственной к заданной алгебре Ли. При этом гамильтониан имеет вид

$$
\mathcal{H}([u], B)=\frac{1}{2}\left\langle[u], I^{-1}[u]\right\rangle+\frac{1}{2}\langle I(B), B\rangle .
$$


Полагая $I(B)=[b]$, получаем следующую формулировку:

$$
[\dot{u}]+L_{I^{-1}[u]}[u]-L_{B}[b]=0, \quad \dot{B}+\left[I^{-1}[u], B\right]=0 .
$$

Можно проверить, что эти уравнения эквивалентны следующему:

$$
([\dot{u}], \dot{B})+\operatorname{ad}_{\left(I^{-1}[u], I(B)\right)}^{*}([u], B)=0 .
$$

Из приведенного выше выражения для гамильтониана $\mathcal{H}$ нетрудно получить уравнения

$$
\frac{\delta \mathcal{H}}{\delta[u]}=I^{-1}[u], \quad \frac{\delta \mathcal{H}}{\delta B}=I(B) .
$$

Тогда можно переформулировать уравнение МГД (13) как

$$
([\dot{u}], \dot{B})+\operatorname{ad}_{(\delta \mathcal{H} / \delta[u], \delta \mathcal{H} / \delta B)}^{*}([u], B)=0
$$

или, в терминах скобки Пуассона, как $([\dot{u}], \dot{B})+\{\mathcal{H},([u], B)\}=0$. Это гамильтоново уравнение на пространстве $S \operatorname{Vect}(\mathcal{X}) \times \Omega^{1}(\mathcal{X}) / Z^{1}(\mathcal{X})$, которое рассматривается как регулярное двойственное пространство магнитного расширения $T^{*} S \operatorname{Vect}(\mathcal{X})$ с гамильтонианом $\mathcal{H}$, определенным выше.

Приведем теперь наш основой результат, показывающий, как эти уравнения МГД деформируются после двойного расширения $T^{*} \operatorname{SVect}(\mathcal{X})$ при условии, что гамильтониан (динамика) не модифицируется. Воспользуемся уравнениями (12), перенесенными на $T^{*} \operatorname{SVect}(\mathcal{X})$ с помощью кинематического изоморфизма:

$$
([\dot{u}], \dot{B})+\widetilde{\operatorname{ad}}_{\left(\left(I^{-1}[u], I(B)\right), \lambda \delta+(U, W)\right)}^{*}(([u], B),(Z, z))=0 .
$$

Теорема 2. Следующая система является гамильтоновой на двойственном пространстве магнитного расширения $T^{*} \operatorname{SVect}(\mathcal{X})$ с гамилътонианом $\mathcal{H}$, определенным в формуле (11):

$$
\begin{aligned}
{[\dot{u}]+L_{\xi}[u]-L_{B}[b]+i_{\xi}[H]+i_{B}[h]+L_{U}[u] } & =0, \\
\dot{B}+[\xi, B]+\xi \wedge h^{\sharp}+\lambda B+[U, B]+[W, \xi] & =0 .
\end{aligned}
$$

ЗАМЕчАНИЕ 1. Мы получили, что $Z=1$ и $z=1$, введя центральные заряды как коэффициенты 2-форм $H$ и $h$. Напомним, что $I^{-1}[u]=\xi$ и $i_{h^{\sharp}}(\omega)=h$. При этом 2-форма $d u$ интерпретируется как завихренность жидкости [10], [12].

Уравнения (14) можно переписать в более сжатом виде:

$$
\begin{gathered}
{[\dot{u}]+L_{\xi+U}[u]-L_{B}[b]+i_{B}[h]+i_{\xi}[H]=0,} \\
\dot{B}+[\xi+U, B-W]+\xi \wedge h^{\sharp}+\lambda B=0 .
\end{gathered}
$$

Напомним, что $[U]$ и $[W]$ являются классами векторных полей в факторе пространства $\overline{S V e c t}(\mathcal{X})$ по $\operatorname{SVect}(\mathcal{X})$, поэтому $[U, W]=0$.

Можно также дать чисто контравариантную формулировку, более близкую к физическому смыслу уравнений МГД:

$$
\begin{array}{r}
\dot{\xi}+\nabla_{\xi} \xi+\nabla p-\operatorname{rot}(B) \wedge B+\xi \wedge H^{\sharp}+B \wedge h^{\sharp}+[U, \xi]=0, \\
\dot{B}+[\xi, B]+\xi \wedge h^{\sharp}+\lambda B+[U, B]+[W, \xi]=0 .
\end{array}
$$


Некоторые частные случаи. В случае тривиальной топологии, т. е. когда $H_{d R}^{1}(\mathcal{X})=H_{d R}^{2}(\mathcal{X})=0$, приведенная выше система редуцируется к следующей:

$$
\dot{\xi}+\nabla_{\xi} \xi+\nabla p-\operatorname{rot}(B) \wedge B=0, \quad \dot{B}+[\xi, B]+\lambda B=0 .
$$

Наличие члена $\lambda B$ изменяет решение: вместо $B$ мы имеем $e^{-\lambda t} B$, поэтому при $\lambda>0$ получается нечто вроде затухающего члена.

Теперь предположим, что $H_{d R}^{1}(\mathcal{X})$ и $H_{d R}^{2}(\mathcal{X})$ являются нетривиальными, тогда присутствие членов $i_{\xi}[H]$ или $\xi \wedge h^{\sharp}$ можно интерпретировать как введение постоянного по времени внешнего магнитного поля $H$. Для чисто гидродинамического случая (т. е. при $B=0$ ) такое уравнение было рассмотрено в книге [10] как уравнение для электронного газа с бесконечной проводимостью. Чтобы как-то интерпретировать члены с внешним дифференцированием, предположим, что $\mathcal{X}=\mathbb{T}^{3}$. Тогда фактор $\overline{\operatorname{SVect}}(\mathcal{X}) / S \operatorname{Vect}(\mathcal{X})$ является трехмерным и порождается классами векторных полей $\partial / \partial x, \partial / \partial y$ и $\partial / \partial z$. Положим, например, $U=\partial / \partial x$ и $W=\partial / \partial y$, тогда уравнения (15) можно переписать в контравариантном виде как

$$
\begin{array}{r}
\dot{\xi}+\nabla_{\xi} \xi+\nabla p-\operatorname{rot}(B) \wedge B+\xi \wedge H^{\sharp}+B \wedge h^{\sharp}+\frac{\partial \xi}{\partial x}=0, \\
\dot{B}+[\xi, B]+\xi \wedge h^{\sharp}+\lambda B+\frac{\partial B}{\partial x}+\frac{\partial \xi}{\partial y}=0
\end{array}
$$

или как

$$
\begin{array}{r}
\left(\frac{\partial}{\partial t}+\frac{\partial}{\partial x}\right) \xi+\nabla_{\xi} \xi+\nabla p-\operatorname{rot}(B) \wedge B+\xi \wedge H^{\sharp}+B \wedge h^{\sharp}=0, \\
\left(\frac{\partial}{\partial t}+\frac{\partial}{\partial x}\right) B+[\xi, B]+\xi \wedge h^{\sharp}+\lambda B+\frac{\partial \xi}{\partial y}=0 .
\end{array}
$$

Таким образом, члены, соответствующие внешним дифференцированиям, индуцируют некоторые изменения времени, как если бы мы сделали замену $t \rightarrow t+x$. Этот факт может, вероятно, иметь релятивистскую интерпретацию.

ЗАмЕчАниЕ 2. Можно сделать еще один шаг, применив теорию групп к симплектической геометрии. Все наши пуассоновы структуры являются структурами Пуассона-Ли на двойственной алгебре Ли. Хорошо известно, что линейную пуассонову структуру на $\mathfrak{g}^{*}$ можно получить из канонической симплектической структуры Лиувилля на кокасательной группе $T^{*} G$ путем симплектической редукции, ассоциированной с естественным действием группы $G$ трансляциями (левыми или правыми), как поясняется в книге [19]. Поэтому с нашей группой, которая интегрирует двойное расширение $\mathcal{G}=T^{(1)} \widehat{\widehat{\operatorname{Siff}}}(\mathcal{X})$, ассоциирована ее кокасательная группа $T^{*} \mathcal{G}$, и нашу динамическую систему можно поднять до $T^{*} \mathcal{G}$ как гамильтонову систему для канонической симплектической структуры. Более того, как было доказано в книге [10], соответствующий гамильтониан $\mathcal{H}$ естественным образом определяет метрику на группе, для которой наше уравнение является уравнением Эйлера, в точности как для МГД.

\section{9. СУПЕРСИММЕТРИЧНЫЙ АНАЛОГ}

Основные определения и конструкции, касающиеся супералгебр Ли, приведены в монографии [23], а некоторые представления о супергеометрии - в прекрасной книге [24]. Мы будем описывать суперверсию алгебр Каца-Муди, впервые построенную В. Кацем. 
С любой алгеброй Ли $\mathfrak{g}$ можно ассоциировать супералгебру Ли $\overline{\mathfrak{g}}$, определяемую следующим образом. Рассмотрим сначала $\mathfrak{g}_{\theta}=\mathfrak{g} \otimes \Lambda(\theta)$, где $\theta$ - нечетная переменная, тогда скобка естественным образом определяется как

$$
[X+\theta x, Y+\theta y]=[X, Y]+\theta[x, Y]+\theta[X, y] .
$$

Поскольку $\overline{\mathfrak{g}}$ и $\mathfrak{g}_{\theta} \oplus \mathbb{K} \delta$ совпадают как $\mathbb{K}$-векторные пространства, скобки с $\delta$ равны $[X, \delta]=0$ и $[\delta, \theta x]=x$. Другими словами, $\overline{\mathfrak{g}}_{0}=\mathfrak{g}, \overline{\mathfrak{g}}_{1}=\mathfrak{g}_{\theta} \oplus \mathbb{K} \delta$, и в супергеометрических терминах $\delta$ действует как $\partial / \partial \theta$. Если теперь алгебра $\mathfrak{g}$ является полупростой с формой Киллинга $\kappa$, то можно повторить конструкцию центрального коцикла Каца-Муди в данном градуированном контексте, где интеграл есть интеграл Березина Ber [24]. Тогда получаем

$$
c(u, v)=\int_{0 \mid 1} \kappa(u, \delta(v)) \operatorname{Ber}
$$

и, если $u=X+\theta x$ и $v=Y+\theta y$, в более явном виде $c(u, v)=\kappa(x, y)$. Данный 2-коцикл является коциклом нечетно-нечетного типа, откуда и следует его симметрия, с первого взгляда кажущаяся удивительной. Таким образом, окончательно получаем двойное расширение, как и в четном случае:

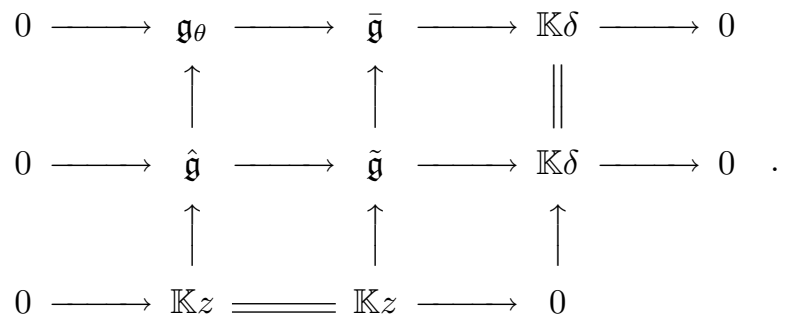

Более того, форма Киллинга естественным образом расширяется на $\hat{\mathfrak{g}}$ как

$$
\hat{\kappa}(u, v)=\int_{0 \mid 1} \kappa(u, v) \text { Ber, }
$$

что дает $\hat{\kappa}(u, v)=\kappa(X, y)+\kappa(x, Y)$, если, как и выше, $u=X+\theta x, v=Y+\theta y$. Пусть $\hat{\kappa}(\delta, z)=1$, а другие члены равны нулю. Это позволяет отождествить $\hat{\mathfrak{g}}$ с ее двойственным пространством. Нечетная и четная части также легко отождествляются: $\hat{\mathfrak{g}}_{0}=\mathfrak{g} \oplus \mathbb{K} z$, а $\hat{\mathfrak{g}}_{1}=\mathfrak{g}_{\theta} \oplus \mathbb{K} \delta$, так что размерности нечетной и четной частей равны, и, таким образом, удовлетворяется одна из главных аксиом суперсимметрии [25].

Можно попытаться построить обобщение на высшие размерности. Пусть $\mathcal{A}_{N}-$ внешняя алгебра на $N$ генераторах и пусть $\mathfrak{g}_{N}=\mathfrak{g} \otimes \mathcal{A}_{N}$. Имеем

$$
H^{1}\left(\mathfrak{g}_{N}, \mathfrak{g}_{N}\right)=\operatorname{Der}\left(\mathcal{A}_{N}\right)=\operatorname{Vect}(0 \mid N) .
$$

Первое равенство представляет собой частный случай формулы (3), второе получается из геометрической интерпретации $\mathcal{A}_{N}$ как алгебры функций на суперпространстве размерности $0 \mid N$, причем его супердифференцирования задаются супералгеброй Ли векторных полей на том же суперпространстве. Здесь следует отметить, что супералгебра Ли $\operatorname{Vect}(0 \mid N)$, называемая алгеброй Ричардсона-Нийенхейса, играет ключевую роль в теории алгебраических деформаций. Для центральных расширений можно воспользоваться градуированным вариантом формулы (4):

$$
H_{2}\left(\mathfrak{g}_{N}\right)=H C_{1}\left(\mathcal{A}_{N}\right)=\Omega^{1}\left(\mathcal{A}_{N}\right) / d \mathcal{A}_{N} .
$$


Таким образом, для $\mathfrak{g}_{N}$ можно построить центральное расширение и расширение с помощью дифференцирований:

$$
\begin{gathered}
0 \longrightarrow \Omega^{1}\left(\mathcal{A}_{N}\right) / d \mathcal{A}_{N} \stackrel{i}{\longrightarrow} \hat{\mathfrak{g}}_{N} \stackrel{\pi}{\longrightarrow} \mathfrak{g}_{N} \longrightarrow 0, \\
0 \longrightarrow \mathfrak{g}_{N} \stackrel{i}{\longrightarrow} \overline{\mathfrak{g}}_{N} \longrightarrow \frac{\pi}{\longrightarrow} \operatorname{Vect}(0 \mid N) \longrightarrow 0
\end{gathered}
$$

В работе [26] центральные расширения алгебры $\mathfrak{g}_{N}$ вычисляются вручную. Мы дадим им интерпретацию, используя двойственный вариант $H^{2}\left(\mathfrak{g}_{N}\right)=H C^{1}\left(\mathcal{A}_{N}\right)$ формулы (4). При этом можно вычислить циклические когомологии, используя последовательность Конна:

$$
H C^{1}\left(\mathcal{A}_{N}\right)=\operatorname{ker} \partial: H H^{1}\left(\mathcal{A}_{N}\right) \rightarrow H H^{0}\left(\mathcal{A}_{N}\right) .
$$

Здесь $H H-$ когомологии Хохшильда, которые равны $H H^{1}\left(\mathcal{A}_{N}\right)=\operatorname{Vect}(0 \mid N)$ и $H H^{0}\left(\mathcal{A}_{N}\right)=\mathcal{A}_{N}$. Вычисления, проведенные в работе [26], позволяют отождествить оператор $\partial$ с супердивергенцией супервекторных полей, откуда получаем изоморфизм $H C^{1}\left(\mathcal{A}_{N}\right)$ с супералгеброй $S \operatorname{Vect}(0 \mid N)$ векторных полей в размерности $0 \mid N$ с нулевой супердивергенцией. Заметим, что имеется двойственность между циклическими гомологиями и когомологиями:

$$
\begin{aligned}
\mathcal{A}_{N}=H H_{0}\left(\mathcal{A}_{N}\right) \stackrel{d}{\longrightarrow} \Omega^{1}\left(\mathcal{A}_{N}\right)=H H_{1}\left(\mathcal{A}_{N}\right) \stackrel{\pi}{\longrightarrow} \\
0 \Omega^{1}\left(\mathcal{A}_{N}\right) / d \mathcal{A}_{N}=H C^{1}\left(\mathcal{A}_{N}\right) \longrightarrow 0 \\
0 \stackrel{i}{\longrightarrow} \operatorname{Sect}(0 \mid N)=H C^{1}\left(\mathcal{A}_{N}\right) \stackrel{i}{\longrightarrow} \operatorname{Vect}(0 \mid N)=H H^{1}\left(\mathcal{A}_{N}\right) \stackrel{\partial}{\longrightarrow} \mathcal{A}_{N}=H H^{0}\left(\mathcal{A}_{N}\right) .
\end{aligned}
$$

Вычисления представляют собой прямое обобщение четного случая (см. книгу [6]). Таким образом, мы получаем, на первый взгляд, неплохую двойственность между расширениями (16) и (17), причем второе ограничено на $S \operatorname{Vect}(0 \mid N)$. Однако, к сожалению, условие совместности (2) выполняется, только если дифференцирования коммутируют друг с другом. Поэтому можно надеяться на двойное расширение, обобщающее случай $N=1$, только в случае абелевых подалгебр $\operatorname{SVect}(0 \mid N)$ и соответствующих им двойственных подалгебр в $\Omega^{1}\left(\mathcal{A}_{N}\right) / d \mathcal{A}_{N}$. Принцип суперсимметрии, согласно которому число нечетных и четных размерностей должно быть одинаково, все еще не нарушается.

При кинематической и динамической интерпретации нужно сначала исследовать супергеометрию коприсоединенных орбит этих алгебр. Любопытно отметить, что на эту тему известно немного, за исключением пионерских работ [27], [28].

\section{0. ЗАКЛЮЧИТЕЛЬНЫЕ ЗАМЕЧАНИЯ}

В настоящем разделе мы для краткости положим $S \operatorname{Vect}(\mathcal{X})=\mathfrak{g}$. В работе [29] изучались деформации этой алгебры Ли, однако она оказалась жесткой. Единственным нетривиальным является случай $n=3$, когда $\operatorname{dim} H^{2}(\mathfrak{g}, \mathfrak{g})=1$, и деформация порождается классом когомологий $\Phi$, который в явном виде построен ниже. К сожалению (или к счастью?), инфинитезимальная деформация, определяемая классом $\Phi$, не допускает никакой пролонгации, откуда и следует жесткость. Тем не менее такая инфинитезимальная деформация определяет реальную деформацию на $T \mathfrak{g}$ следующим образом:

$$
[X+t x, Y+t y]=[X, Y]+t[x, Y]+t[X, y]+t \lambda \Phi(X, Y)
$$


где скалярный параметр $\lambda$ произволен. Напомним конструкцию класса когомологий $\Phi$ из работы [29]. Имеем точную последовательность

$$
0 \longrightarrow \mathfrak{g} \stackrel{i}{\longrightarrow} \Omega^{2}(\mathcal{X}) \stackrel{d}{\longrightarrow} \Omega^{3}(\mathcal{X}) \longrightarrow 0 .
$$

Как и выше, $i(X)=i_{X} \omega, \omega$ - форма объема, а $d$ - внешний дифференциал; точность данной последовательности понимается в смысле теории пучков. Теперь можно привести некоторые соображения по поводу класса $\Phi$. Естественные коциклы на алгебрах Ли векторных полей, обобщающие дивергенцию на высшие степени, были построены Гельфандом [30]. Здесь нам потребуется класс когомологий

$$
\Psi: \operatorname{Vect}(\mathcal{X}) \times \operatorname{Vect}(\mathcal{X}) \rightarrow \Omega^{2}(\mathcal{X}) .
$$

Можно привести локальную формулу, которая прояснит тот факт, что мы действительно обобщили дивергенцию:

$$
\Psi(X, Y)=\partial_{a i} X^{b} \partial_{b j} Y^{a} d x_{i} \wedge d x_{j}
$$

(здесь мы использовали обозначения Эйнштейна для суммирования по индексам).

Формула (18) знакома всем, кто интересуется деформационным квантованием, она представляет собой вариант коцикла Вея - ключевой элемент при построении звездчатого произведения. Теперь можно построить $\Phi$, ограничивая $\Psi$ на $\mathfrak{g}$, а затем беря свертку с тензором объема, двойственным $\omega$. Здесь мы приводим только основные идеи, полное доказательство имеет гораздо более технический характер [29]. Можно также распространить эту деформацию на магнитное расширение $T^{*} \mathfrak{g}$, используя кинематический изоморфизм $\mathcal{J}^{\sharp}$; это было проделано в работе [31], где получен коцикл вида $\tau: \mathfrak{g} \times \mathfrak{g} \rightarrow \Omega^{1}(\mathcal{X}) / Z^{1}(\mathcal{X})$, локально определяемый как $\tau(X, Y) \mid=\left[\partial_{a} X^{b} d\left(\partial_{b} Y^{a}\right)\right]$. Этот результат автор работы [31] связывает с результатами своих ранних работ по абелевым (т. е. нецентральным) расширениям [2], которые называются тороидальными алгебрами Ли. Кроме того, он подчеркивает аналогию с коциклом Вирасоро, который также имеет дифференциальный порядок 3.

На двойственной к деформированной алгебре Ли $T^{*} \mathfrak{g}_{\tau}$ уравнения МГД естественным образом деформируются как новая гамильтонова система:

$$
([\dot{u}], \dot{B})+\operatorname{ad}_{(\tau)\left(I^{-1}[u], I(B)\right)}^{*}([u], B)=0 .
$$

В контравариантных терминах деформированные уравнения имеют вид

$$
\dot{\xi}+\nabla_{\xi} \xi+\nabla p-\operatorname{rot}(B) \wedge B+\operatorname{Div} \mathcal{T}=0, \quad \dot{B}+[\xi, B]=0,
$$

где $\mathcal{T}$ с физической точки зрения интерпретируется как асимметричный тензор напряжений,

$$
\mathcal{T}^{i j}=\frac{\partial B^{j}}{\partial x_{a}} \frac{\partial \xi^{a}}{\partial x_{i}}
$$

который появляется в уравнениях через деформированный коцикл $\tau$ (см. подробности в работе [2]). 


\section{ПРИЛОЖЕНИЕ}

Докажем, что $H C_{1}(\mathcal{A})=0$, если $\mathcal{A}=\mathbb{K}[t] /\left(t^{2}\right)$ - локальное кольцо дуальных чисел. Следуя работе [7], можно найти кэлеровы 1-формы в явном виде. Пусть $I=\operatorname{ker} \mu: \mathcal{A} \otimes \mathcal{A} \rightarrow \mathcal{A}$. Это ядро двумерно и имеет генераторы $t \otimes t$ и $1 \otimes t-t \otimes 1$. Тогда $I^{2}$ порождается элементом $t \otimes t$, а пространство $\Omega^{1}(\mathcal{A})=I / I^{2}$ одномерно, причем $d: \mathcal{A} \rightarrow \Omega^{1}(\mathcal{A})$ - изоморфизм. Поэтому $H C_{1}(\mathcal{A})=\Omega^{1}(\mathcal{A}) / d \mathcal{A}=0$. На самом деле это доказательство можно обобщить на случай любых артиновых колец усеченных многочленов $\mathcal{A}_{k}=\mathbb{K}[t] /\left(t^{k}\right)$ (см. работу [6]).

Благодарности. Автор благодарен всем, кто вел с ним полезные обсуждения в процессе выполнения настоящей работы, особенно Борису Хесину и Тудору Ратьу, а также Корнелию Визману и Гейсу Тейнману за их полезные указания. Автор также благодарен Алисе Фиаловски и Анне Пишро за приглашение выступить с докладом на эту тему на конференциях в Будапеште и Сент-Этьене.

\section{Список литературы}

[1] P. J. Hilton, U. Stammbach, A Course in Homological Algebra, Graduate Texts in Mathematics, 4, Springer, New York, 1997.

[2] Y. Billig, Lett. Math. Phys., 64:2 (2003), 155-169.

[3] A. Haddi, Commun. Algebra, 20:4 (1992), 1145-1166.

[4] A. Haddi, C. R. Acad. Sci. Paris. Sér. I Math., 306:16 (1988), 691-694.

[5] P. Zusmanovich, Astérisque, 226 (1994), 435-452.

[6] J.-L. Loday, Cyclic Homology, Grundlehren der Mathematischen Wissenschaften, 301, Springer, Berlin, 1998.

[7] R. Hartshorne, Algebraic Geometry, Graduate Texts in Mathematics, 52, Springer, New York, 1977.

[8] V.C. Kac, Infinite-Dimensional Lie Algebras. An introduction, Progress in Mathematics, 44, Birkhäuser, Boston, MA, 1983.

[9] A. A. Kirillov, Lectures on the Orbit Method, Graduate Studies in Mathematics, 64, AMS, Providence, RI, 2004.

[10] V. Arnold, B. A. Khesin, Topological Methods in Hydrodynamics, Applied Mathematical Sciences, 125, Springer, New York, 1998.

[11] B. Khesin, R. Wendt, The Geometry of Infinite-Dimensional Groups, Ergebnisse der Mathematik und ihrer Grenzgebiete. 3 Folge, 51, Springer, Berlin, 2009.

[12] B. Khesin, Symplectic structures and dynamics on vortex membranes, arXiv: 1201.5914.

[13] A. Lichnerowicz, Ann. Inst. Fourier (Grenoble), 24:3 (1974), 219-266.

[14] C. Vizman, Ann. Univ. Vest Timiş. Ser. Mat.-Inform., 48:1-2 (2010), 285-297.

[15] S. Haller, C. Vizman, Math. Ann., 329:4 (2004), 771-785.

[16] C. Roger, "The group of volume preserving diffeomorphisms and the Lie algebra of unimodular vector fields: survey of some classical and not-so-classical results", Twenty Years of Bialowieza: a Mathematical Anthology, World Scientific Monograph Series in Mathematics, 8, eds. S. T. Ali, G. G. Emch, A. Odzijewicz, M. Schlichenmaier, S. L. Woronowicz, World Sci., Hackensack, NJ, 2005, 79-98.

[17] C. Roger, "Unimodular vector fields and deformation quantization", Deformation Quantization, Proceedings of the meeting of theoretical physicists and mathematicians (Strasbourg, France, May 31 -June 2, 2001), IRMA Lectures in Mathematics and Theoretical Physics, 1, ed. G. Halbout, de Gruyter, Berlin, 2002, 135-148.

[18] C. Roger, Rep. Math. Phys., 35:2-3 (1995), 225-266. 
[19] J.E. Marsden, T.S. Ratiu, Introduction to Mechanics and Symmetry. A Basic Exposition of Classical Mechanical Systems, Texts in Applied Mathematics, 17, Springer, New York, 1999.

[20] A. Banyaga, The Structure of Classical Diffeomorphism Groups, Mathematics and its Applications, 400, Kluwer, Dordrecht, 1997.

[21] V. Ovsienko, C. Roger, Comm. Math. Phys., 273:2 (2007), 357-378, arXiv: math-ph/0602043.

[22] D. Biskamp, Nonlinear Magnetohydrodynamics, Cambridge Monographs on Plasma Physics, 1, Cambridge Univ. Press, Cambridge, 1993.

[23] M. Scheunert, The Theory of Lie Superalgebras. An introduction, Lecture Notes in Mathematics, 716, Springer, Berlin, 1979.

[24] P. Deligne, P. Etingof, D. S. Freed, L. C. Jeffrey, D. Kazhdan, J. W. Morgan, D. R. Morrison, E. Witten (eds.), "Quantum Fields and Strings: A Course for Mathematicians", v. 1, 2, Material from the Special Year on Quantum Field Theory (Princeton, NJ, 1996-1997), AMS, Providence, RI, 1999.

[25] П. Уэст, Введение в суперсимметрию и супергравитацию, Мир, М., 1989.

[26] R. Coquereaux, L. Frappat, E. Ragoucy, P. Sorba, Comm. Math. Phys., 133:1 (1990), 1-35.

[27] G. M. Tuynman, J. Geom. Phys., 60:12 (2010), 1919-1939.

[28] G. M. Tuynman, Geometric quantization of superorbits: a case study, arXiv: 0901.1811.

[29] P. B. A. Lecomte, C. Roger, J. Differential Geom., 44:3 (1996), 529-549.

[30] I. M. Gel'fand, "The cohomology of infinite dimensional Lie algebras: some questions of integral geometry", Actes du Congrès International des Mathématiciens, v. 1 (Nice, France, 1970), Gauthier-Villars, Paris, 1971, 95-111.

[31] Y. Billig, J. Math. Phys., 46:4 (2005), 043101, 13 pp., arXiv: math-ph/0401052.

Поступила в редакцию 12.06.2012 